\title{
PAPER
}

\section{Subdural haematoma: a potentially serious consequence of spontaneous intracranial hypotension}

\section{R J de Noronha, B Sharrack, M Hadjivassiliou, C A J Romanowski}

J Neurol Neurosurg Psychiatry 2003;74:752-755

See end of article for authors' affiliations

....................

Correspondence to: Dr C A J Romanowski, Department of Radiology, Royal Hallamshire Hospital, Glossop Road, Sheffield S10 2FJ, UK: charles.romanowski@ sth.nhs.uk

Received 26 June 2001 In final revised form 8 January 2003

Accepted

27 January 2003
Background: Spontaneous intracranial hypotension $(\mathrm{SIH})$ is characterised by postural headache and low opening pressure at lumbar puncture without obvious cause. Cranial magnetic resonance imaging often shows small subdural collections without mass effect, dural enhancement, venous sinus dilatation, or downward displacement of the brain. The condition is thought to be benign.

Objectives: To evaluate the incidence of subdural haematoma as a serious complication of $\mathrm{SIH}$.

Methods: A prospective survey of all cases of $\mathrm{SIH}$ presenting to a large neuroscience unit over a two year period.

Results: Nine cases of SIH were seen. Four of these were complicated by acute clinical deterioration with reduced conscious level because of large subdural haematomas requiring urgent neurosurgical drainage.

Conclusions: SIH should not be considered a benign condition. Acute deterioration of patients' clinical status may occur secondary to large subdural haematomas, requiring urgent neurosurgical intervention.
$\mathrm{T}$ he syndrome of intracranial hypotension is characterised by postural headache and low opening pressure at lumbar puncture. Spontaneous intracranial hypotension (SIH) was first described by Schaltenbrand in 1938 and was thought to be a rare condition. ${ }^{1}$ The advent of magnetic resonance imaging (MRI) has led to an increase in the diagnosis of this condition, with many recent reports detailing the clinical and the radiological features and the management..$^{2-6}$ Typical cranial MRI features are small subdural collections without mass effect, dural enhancement, venous sinus dilatation, or downward displacement of the brain. ${ }^{2578}$ When a lumbar puncture is done, the opening pressure is low (less than $6 \mathrm{~cm} /$ $\left.\mathrm{H}_{2} \mathrm{O}\right){ }^{4}$ The cerebrospinal fluid (CSF) may contain red and white cells and the protein may be increased. ${ }^{3468}$

The condition is generally considered to be benign, and the majority of patients improve with conservative management. ${ }^{48}$ A review of published reports revealed a few cases of SIH complicated by small subdural collections which required neurosurgical drainage..$^{9-14}$ However, there are no records of large subdural haematomas causing a decreased conscious level and requiring urgent neurosurgical drainage. This study was undertaken to assess the incidence of this serious complication of SIH.

\section{METHODS}

We carried out a prospective survey of all patients with SIH who presented to our institution-a regional neuroscience centre serving a catchment population of 2.2 millionbetween 1999 and 2000. Nine patients with SIH (three women and six men, median age 39 years, range 23 to 66) were seen during this period. The diagnosis of SIH was made on the basis of the history, clinical examination, low CSF pressure at lumbar puncture, and the cranial MRI appearances.

\section{RESULTS}

Of the nine patients with SIH, four experienced acute deterioration with increasing severity of headache, focal neurological deficits, and impaired consciousness. On reimaging, all four patients were found to have large subdural haematomas with mass effect which required urgent neurosurgical drainage. The radiological signs present on the initial cranial MRI in these four patients are listed in table 1 . Their clinical histories are summarised below.

\section{Case 1}

A 66 year old man presented with sudden onset of cervical pain radiating to the occiput and associated with neck stiffness. Except for a slightly unsteady gait, neurological examination was normal. Cranial computed tomography (CT) done four days after the onset of symptoms was normal. At lumbar puncture the opening pressure was $5 \mathrm{~cm} / \mathrm{H}_{2} \mathrm{O}$ and the CSF was uniformly blood stained. A presumptive diagnosis of subarachnoid haemorrhage was made and the patient underwent a cerebral angiogram, which was normal. The symptoms

Table 1 The radiological signs present on initial cranial magnetic resonance imaging, showing signs of spontaneous intracranial hypotension

\begin{tabular}{|c|c|c|c|c|c|c|c|}
\hline Case & $\begin{array}{l}\text { Age } \\
\text { (years) }\end{array}$ & $\begin{array}{l}\text { Iter } \\
\text { displacement* }\end{array}$ & $\begin{array}{l}\text { Tonsil } \\
\text { displacement }\end{array}$ & $\begin{array}{l}\text { Dural } \\
\text { enhancement }\end{array}$ & Sinus & Collection depth & Site of subdural collection \\
\hline 1 & 66 & $-3 \mathrm{~mm}$ & $-3 \mathrm{~mm}$ & Enhancement & Sag sinus dilated & Left $4 \mathrm{~mm}$; right $4 \mathrm{~mm}$ & Supra- and infratentorial \\
\hline 2 & 49 & $-8 \mathrm{~mm}$ & $+2 \mathrm{~mm}$ & No contrast & Sag sinus dilated & Left $8 \mathrm{~mm}$; right $5 \mathrm{~mm}$ & Supra- and infratentorial \\
\hline 3 & 39 & $-10 \mathrm{~mm}$ & $0 \mathrm{~mm}$ & No contrast & Sag sinus dilated & Left $8 \mathrm{~mm}$; right $8 \mathrm{~mm}$ & Supra- and infratentorial \\
\hline
\end{tabular}

Case 4 did not have radiological demonstration of spontaneous intracranial hypotension before development of subdural haematoma. ${ }^{*}$ Displacement of iter as defined by Reich.[15] 


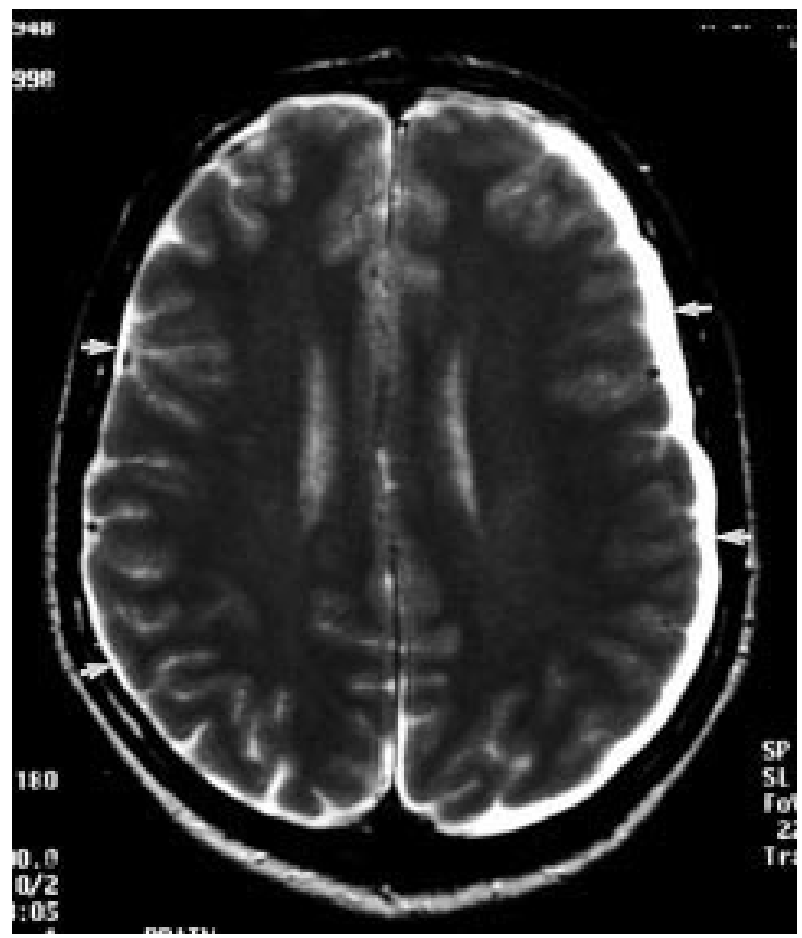

Figure 1 Case 2: axial T2 weighted cranial magnetic resonance imaging, showing bilateral subdural collections, larger on the left than on the right (white arrows).

worsened, but repeat cranial CT was normal. Cranial MRI was done 16 days after the onset of symptoms. This showed bilateral small subdural collections over both cerebral and cerebellar hemispheres, extending over the surface of the clivus, with downward displacement of the brain and flattening of the optic pathways. There was marked dural enhancement following the administration of gadolinium. A diagnosis of SIH was made and the classic history of a low pressure headache was elicited retrospectively. The patient was treated symptomatically and discharged home with only a mild residual headache. One month later he returned with an increasingly severe and constant headache, confusion, and subjective weakness in both legs and the left hand. He became progressively confused and drowsy. Cranial CT showed bilateral large isodense subdural collections with mass effect and a small area of acute haemorrhage in the right subdural space. Urgent drainage of the subdural collections (which were found to be consistent with chronic subdural haematomas) was undertaken through bilateral burr holes. The patient made an uneventful recovery and remained well.

\section{Case 2}

A previously fit 49 year old man developed sudden onset of low pressure headache radiating to the neck, associated with diplopia, photophobia, and nausea. Other than frequent weight training, he had experienced no trauma. The symptoms progressed gradually and by day 11 of his illness he sought medical help. Cranial CT was normal. Cranial MRI showed bilateral thin subdural collections, larger on the left (fig 1), with small infratentorial collections, flattening of the optic pathways, and depression of the iter. Lumbar puncture was done after the MRI, but the CSF pressure was not measured at the referring hospital. A diagnosis of spontaneous intracranial hypotension was made on the basis of the MRI and the clinical findings, and the patient was treated symptomatically and discharged home. Seven weeks later he presented again with worsening headache. His condition deteriorated rapidly following admission, with progressive

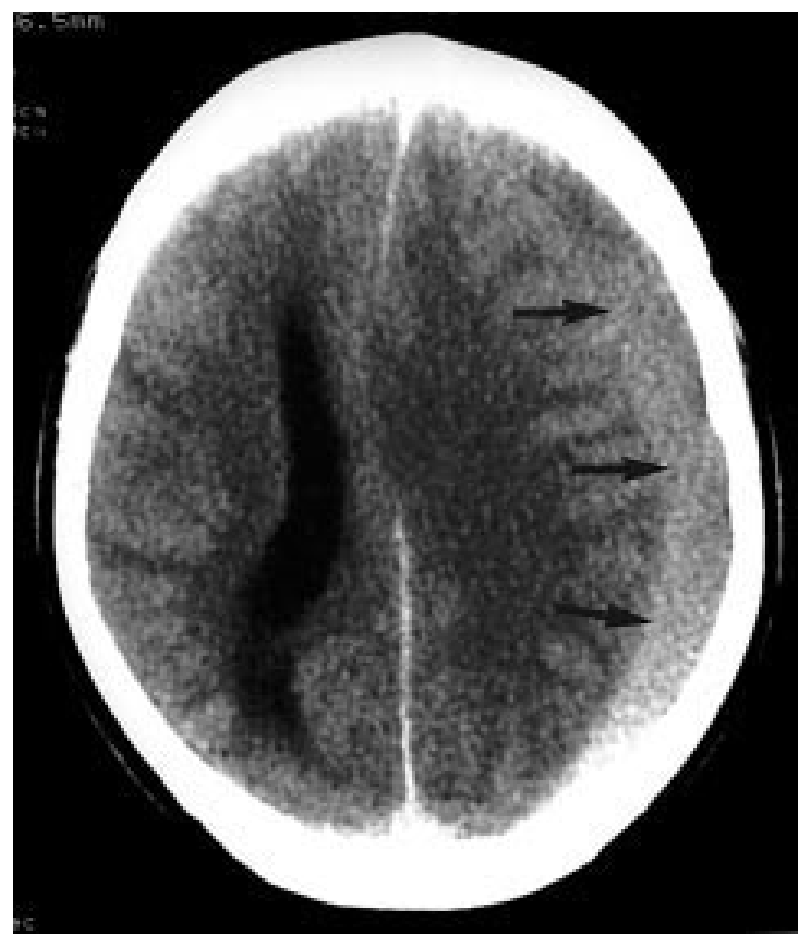

Figure 2 Case 2: cranial computed tomography, showing a left sided isodense subdural collection (black arrows) with midline shift and secondary hydrocephalus of the right lateral ventricle.

drowsiness. Cranial CT showed a left sided isodense subdural collection with midline shift and secondary hydrocephalus of the right lateral ventricle (fig 2). Blood coagulation was normal. His condition continued to deteriorate and he required ventilation before transfer to our neurosurgical unit where his chronic subdural haematoma was drained. He made a good postoperative recovery but required two additional operations to drain recurrent subdural collections. Six months later he remained well.

\section{Case 3}

A previously fit 39 year old man presented with a 10 week history of low pressure headache which started acutely while he was playing golf. Cranial MRI showed bilateral subdural collections overlying both hemispheres and the undersurface of the tentorium, extending down over the clivus and the upper cervical spinal canal. There was some brain stem descent and flattening of the optic chiasm (fig 3). He was treated with an epidural blood patch. Three days later his condition deteriorated, with increasing headache and mild confusion. There were no focal signs on neurological examination. Cranial CT showed bilateral isodense subdural collections, larger on the left, with effacement of the third ventricle and cortical sulci. His condition continued to deteriorate, with increasing drowsiness, neck stiffness, focal motor seizures of the right arm, and sluggish pupillary light reflexes. Repeat cranial CT showed acute subdural bleeding on the left side and compression of the third ventricle and perimesencephalic cisterns. Subdural haematomas were drained through bilateral burr holes. Postoperatively, he remained ataxic and dysarthric. Repeat MRI showed evidence of small petechial haemorrhages at the ponto-mesencephalic junction, presumably representing small Duret haemorrhages resulting from brain stem descent (fig 4). His spinal MRI was normal. Six months after surgery, he was improving gradually.

\section{Case 4}

A 39 year old woman presented with a 12 week history of postural low pressure headache and neck pain. Three weeks 


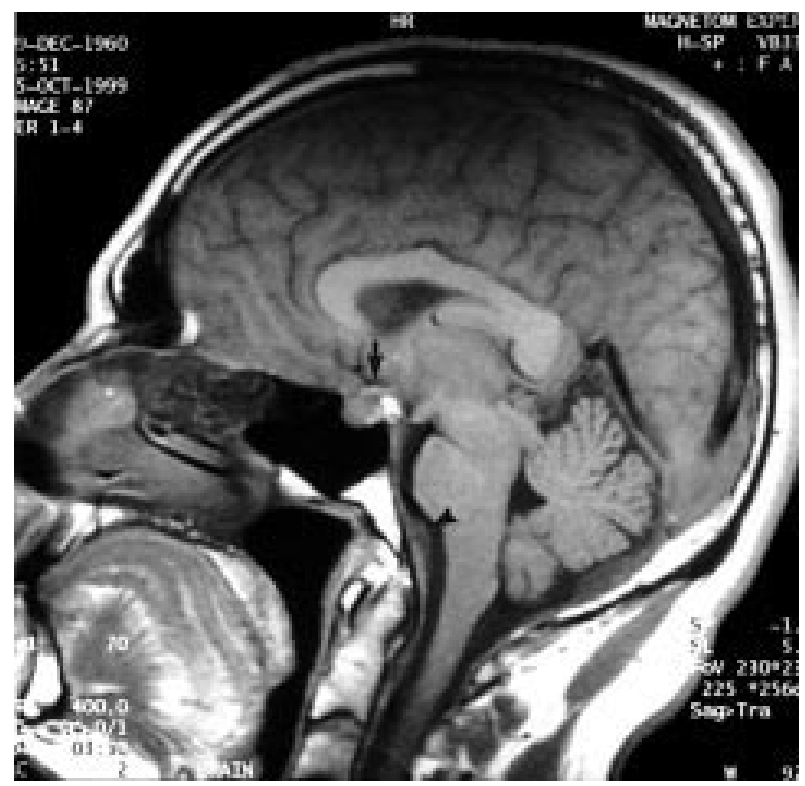

Figure 3 Case 3: sagittal T1 weighted cranial magnetic resonance imaging, showing subdural collections extending down over the clivus (black arrowhead) with brain stem descent (demonstrated by downwards displacement of the iter relative to the incisural line-see Reich $^{15}$ ), and flattening of the optic chiasm (black arrow).

before admission there had been an acute deterioration with vomiting and worsening of the headache, which became constant and was no longer postural. There was no history of trauma, and neurological examination was normal. Blood coagulation was normal. The clinical picture was suggestive of SIH with acute deterioration. Cranial CT showed bilateral large isodense subdural collections, larger on the right side, with a small area of acute haemorrhage within the right subdural space. The ventricles were compressed, with midline shift to the left. Cranial MRI showed bilateral large subdural collections of high proton density and Tl weighted signal, with lower than CSF T2 weighted signal. The collections were surgically drained within 24 hours of presentation and were found to consist of old blood. The patient remained well. Though SIH was not confirmed radiologically in this patient before her clinical deterioration and subsequent neurosurgical drainage of the subdural haematomas, the clinical description of the preceding headaches was characteristic of the condition. No other cause of non-traumatic subdural haematomas could be found.

\section{DISCUSSION}

SIH is generally considered to be a benign condition, with the majority of patients requiring only symptomatic treatment. ${ }^{38}$ However, in some patients symptoms persist, and occlusion of the CSF leak is required for symptomatic control. Epidural blood patch and surgical repair of meningeal diverticula have both been used successfully. ${ }^{3}{ }^{6} 7$ 16-18

Subdural haemorrhage is a rare but recognised complication of intracranial hypotension, occurring secondary to epidural anaesthesia, lumbar puncture, and ventriculoperitoneal shunt. ${ }^{1920}$

The reported incidence of subdural collections in association with SIH is of the order of $10 \% \cdot{ }^{389}$ However, published reports are unclear about the pathological nature of these collections, the terms "collection," "effusion," "hygroma," and "haematoma" being used interchangeably in various papers. $^{348-10}$ In this report, we have opted to use the term "collection" in all our cases except when blood was identified at surgery. A review of the literature has revealed a few case reports of SIH associated with small asymmetrical subdural

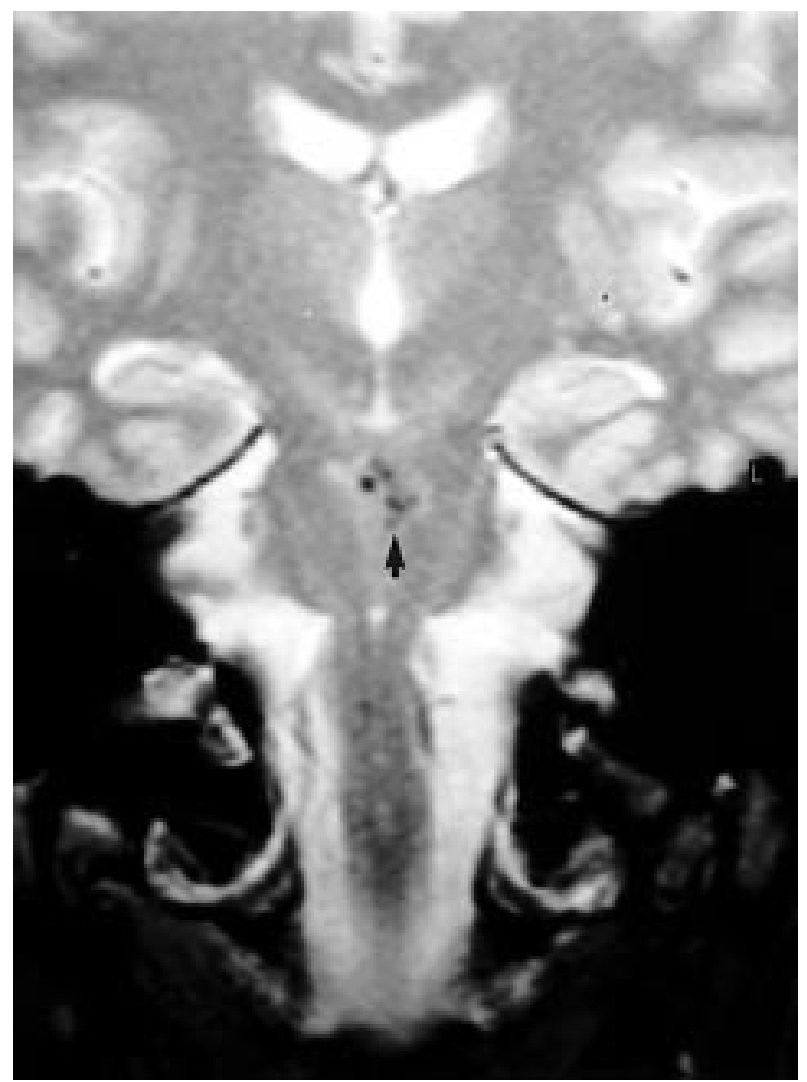

Figure 4 Case 3: coronal gradient echo T2* weighted cranial magnetic resonance imaging, showing small petechial

haemorrhages (black arrow) at the pontomesencephalic junction representing Duret haemorrhages resulting from brain stem descent.

collections, and a total of nine SIH cases complicated by subdural collection which required neurosurgical drainage. ${ }^{4-1421}$ In six of these cases the intracranial pressure was reported to have been low at surgery. ${ }^{510121421}$ The intracranial pressure at the time of neurosurgical intervention in the other cases was not reported, although presurgical cranial MRI in one of the cases showed mass effect and midline shift. ${ }^{13}$ In three cases, patients experienced a decreased conscious level, but the intracranial pressure was not found to be raised at surgery, and the decreased consciousness was thought to reflect low infratentorial pressure and brain descent because of loss of the CSF cushion effect. ${ }^{9}{ }^{121}$ In two cases the development of subdural haematomas was associated with the disappearance of postural headache several weeks after the diagnosis of SIH. This was thought to reflect normalisation of the previously low intracranial pressure. ${ }^{11} \mathrm{~A}$ decreased conscious level in the presence of intracranial hypotension was described in one further case of SIH which did not undergo neurosurgical drainage. The cause was felt to be diencephalic compression secondary to brain descent. ${ }^{18}$

The four patients we describe here all experienced acute deterioration of their symptoms as a result of subdural haemorrhage, associated with mass effect and a midline shift resulting in decreased consciousness. With the development of the subdural haemorrhage, the characteristics of the headache in all four patients changed from being postural to being constant and becoming more severe. In three of the four patients there was also a reduction in the conscious level before neurosurgical intervention. Although intracranial pressure was not formally measured in our patients, the subdural haematomas were found to be under pressure when drained.

We postulate that the presence of small subdural collections in our patients predisposed them to rupture of cortical veins 
crossing the subdural space and the subsequent development of large subdural haematomas. In view of these four cases, and those reviewed above, SIH should not be considered a benign condition. A serious decrease in conscious level may occur secondary to decreased intracranial pressure and brain descent. Our cases further demonstrate that a decreased conscious level can also occur secondary to intracranial haemorrhage and a subsequent rise in intracranial pressure. Differentiating between these two conditions is obviously important as the treatment is different. The management in the former should be directed towards increasing the infratentorial pressure by means of an intrathecal saline infusion or various therapeutic manoeuvres aiming at sealing the CSF leak, whereas in cases of large subdural haemorrhage surgical drainage is required initially, although treatment of the underlying cause of SIH, if identified, may also be required.

Patients with SIH should be advised to seek medical help urgently if their symptoms deteriorate. Prompt imaging of these patients with cranial CT should be advised. The diagnosis of spontaneous intracranial hypotension should be considered in patients presenting with subdural haematoma in the absence of trauma and with normal clotting, particularly as subdural haematomas secondary to intracranial hypotension may recur following drainage, and treatment of the underling cause is required.

\section{Authors' affiliations}

R J de Noronha, C A J Romanowski, Department of Radiology, Royal Hallamshire Hospital, Sheffield Teaching Hospital NHS Trust, Sheffield, UK

B Sharrack, M Hadjivassiliou, Department of Neurology, Royal Hallamshire Hospital

Competing interests: none declared

\section{REFERENCES}

1 Schaltenbrand G. Neure anshaungun zur pathophysiologie der liquorzirkulation. Zentrabl Neurochir 1938;3:290-300.

2 Horton JC, Fishman RA. Neurovisual findings in the syndrome of spontaneous intracranial hypotension from dural cerebrospinal fluid leak. Ophthalmology 1994;101:244-51.
3 Renowden SA, Gregory R, Hyman N, et al. Spontaneous intracranial hypotension. J Neurol Neurosurg Psychiatry 1995;59:511-15.

4 Rando TA, Fishman RA. Spontaneous intracranial hypotension. Neurology 1992:42:481-7.

5 Pannullo SC, Reich JB, Krol G, et al. MRI changes in intracranial hypotension. Neurology 1993;43:919-26.

6 Schievink WI, Meyer FB, Atkinson JL, et al. Spontaneous spinal cerebral fluid leaks and intracranial hypotension. J Neurosurg 1996;84:598605.

7 Fishman RA, Dillon WP. Dural enhancement and cerebral displacement secondary to intracranial hypotension. Neurology 1993;43:609-11

8 Christoforidis GA, Mehta BA, Landi JL, et al. Spontaneous intracranial hypotension: report of four cases and review of the literature. Neuroradiology 1998;40:636-43.

9 Sipe JC, Zyroff J, Waltz TA. Primary intracranial hypotension and bilateral isodense subdural haematomas. Neurology 1981;31:334-7.

10 Nakajima H, Sakai T, Aoki N, et al. Bilateral chronic subdural haematomas associated with intracranial hypotension. Neurol Med Chir 1996;36:647-9.

11 Sato $\mathrm{Y}$, Honda $\mathrm{Y}$, Maruoka $\mathrm{H}$, et al. Subdural haematoma following disappearance of orthostatic headache and pressure normalization in two patients with spontaneous intracranial hypotension. Cephalalgia 1998;18:60-3

12 Aoki N, Sakai T, Oikawa A. Spontaneous intracranial hypotension. Acta Neurochir 1998;140:47-9.

13 Mikawa S, Ebina T. Spontaneous intracranial hypotension complicating subdural hematoma: unilateral oculomotor nerve palsy caused by epidural blood patch. No Shinkei Geka Neurol Surg 2001;29:747-53.

14 Murakami M, Morikawa K, Matsuno A, et al. Spontaneous intracranial hypotension associated with bilateral chronic subdural haematomas. Neurol Med Chir 2000;40:484-8.

15 Reich JB, Sierra J, Camp W, et al. Magnetic resonance imaging measurements and clinical changes accompanying transtentorial and foramen magnum brain herniation. Ann Neurol 1993;33:159-70.

16 Schievink WI, Reimer R, Folger WN. Surgical treatment of spontaneous intracranial hypotension associated with a spinal arachnoid diverticulum. I Neurosurg 1994;80:736-9.

17 Davenport RJ, Chataway SJS, Warlow CP. Spontaneous intracranial hypotension from a CSF leak in a patient with Marfan's syndrome. J Neurol Neurosurg Psychiatry 1995;59:516-19.

18 Pleasure SJ, Abosch A, Friedman J, et al. Spontaneous intracranial hypotension resulting in stupor caused by diencephalic compression. Neurology 1998;50: 1854-7.

19 Jonsson O, Einarsson P, Olsson GL. Subdural haematoma and spinal anaesthesia. Anaesthesia 1983;38:144-6.

20 Vos PE, de Boer WA, Wurzer JA, et al. Subdural haematoma after lumbar puncture. Two case reports and review of the literature. Clin Neurol Neurosurg 1991;93:127-32.

21 Beck CE, Rizk NW, Kiger LT, et al. Intracranial hypotension presenting with severe encephalopathy. J Neurosurg 1998;89:470-3. 